\title{
High-throughput phenotyping-based QTL mapping reveals the genetic architecture of the salt stress tolerance of Brassica napus
}

\author{
Guofang Zhang ${ }^{1}$, Jinzhi Zhou ${ }^{1}$, Yan Peng ${ }^{1}$, Zengdong Tan ${ }^{1}$, Yuting Zhang ${ }^{1}$, Hu Zhao ${ }^{1}$, \\ Dongxu Liu ${ }^{1}$, Xiao Liu ${ }^{1}$, Long Li ${ }^{1}$, Liangqian $\mathrm{Yu}^{1}$, Cheng $\mathrm{Jin}^{1}$, Shuai Fang ${ }^{1}$, Jiawei Shi ${ }^{1}$, \\ Zengdong Geng ${ }^{1}$, Shanjing Yang ${ }^{1}$, Guoxing Chen ${ }^{1}$, Kede Liu ${ }^{1}$, Qingyong Yang ${ }^{1}$, Hui Feng ${ }^{1}$, \\ Liang Guo ${ }^{1}$, and Wanneng Yang ${ }^{1}$ \\ ${ }^{1}$ Huazhong Agricultural University
}

September 25, 2021

\begin{abstract}
Salt stress is a major limiting factor that severely affects the survival and growth of crops. It is important to understand the salt tolerance ability of Brassica napus and explore the underlying related genetic resources. We used a high-throughput phenotyping platform to quantify 2,111 image-based traits (i-traits) of a natural population under 3 different salt stress conditions and an intervarietal substitution line (ISL) population under 9 different stress conditions to monitor and evaluate the salt stress tolerance of B. napus over time. We finally identified 928 high-quality i-traits associated with the salt stress tolerance of B. napus. Moreover, we mapped the salt stress-related loci in the natural population via a genome-wide association study (GWAS) and performed a linkage analysis associated with the ISL population, respectively. The results revealed 234 candidate genes associated with salt stress response, and two novel candidate genes, BnCKX5 and BnERF3, were experimentally verified to regulate the salt stress tolerance of B. napus. This study demonstrates the feasibility of using high-throughput phenotypingbased QTL mapping to accurately and comprehensively quantify i-traits associated with B. napus. The mapped loci could be used for genomics-assisted breeding to genetically improve the salt stress tolerance of B. napus.
\end{abstract}

\section{Hosted file}

Manuscript-PCE.pdf available at https://authorea.com/users/392178/articles/539044-highthroughput-phenotyping-based-qtl-mapping-reveals-the-genetic-architecture-of-the-saltstress-tolerance-of-brassica-napus 